Anal. Chem. Supporting Information

\title{
Chiral Recognition of 2-Alkylalcohols with Magnetic Circular Dichroism Measurement of Porphyrin J-Aggregate on Silica Gel Plate
}

Hitoshi Watarai* and Yuriko Kurahashi

Institute for NanoScience Design, Osaka University, Toyonaka, Osaka 560-8531, Japan

watarai@chem.sci.osaka-u.ac.jp

\section{Abstract}

Simple chiral recognition method of 2-alkylalcohols on silica gel plate was proposed by using the induced circular dichroism (ICD) of J-aggregates of diprotonated tetraphenylporphyrin and magnetic circular dichroism (MCD) spectrometry. To the silica gel on a glass slide including a chiral 2-alkylalcohol and $4 \mathrm{M}$ sulfuric acid, the porphyrin in toluene was added and mixed. Then, the glass slide was used for in situ MCD measurement. The observed ICD spectra could recognize well the chirality of the alcohols and the ICD intensity normalized by the MCD intensity of the J-aggregate correlated linearly to the amount of the chiral alcohols in the silica gel.

1. Measurement of magnetic circular dichroism (MCD) spectra of silica gel plate Figure S-1 shows the schematic illustration of the method of MCD measurements of the glass plate sample.
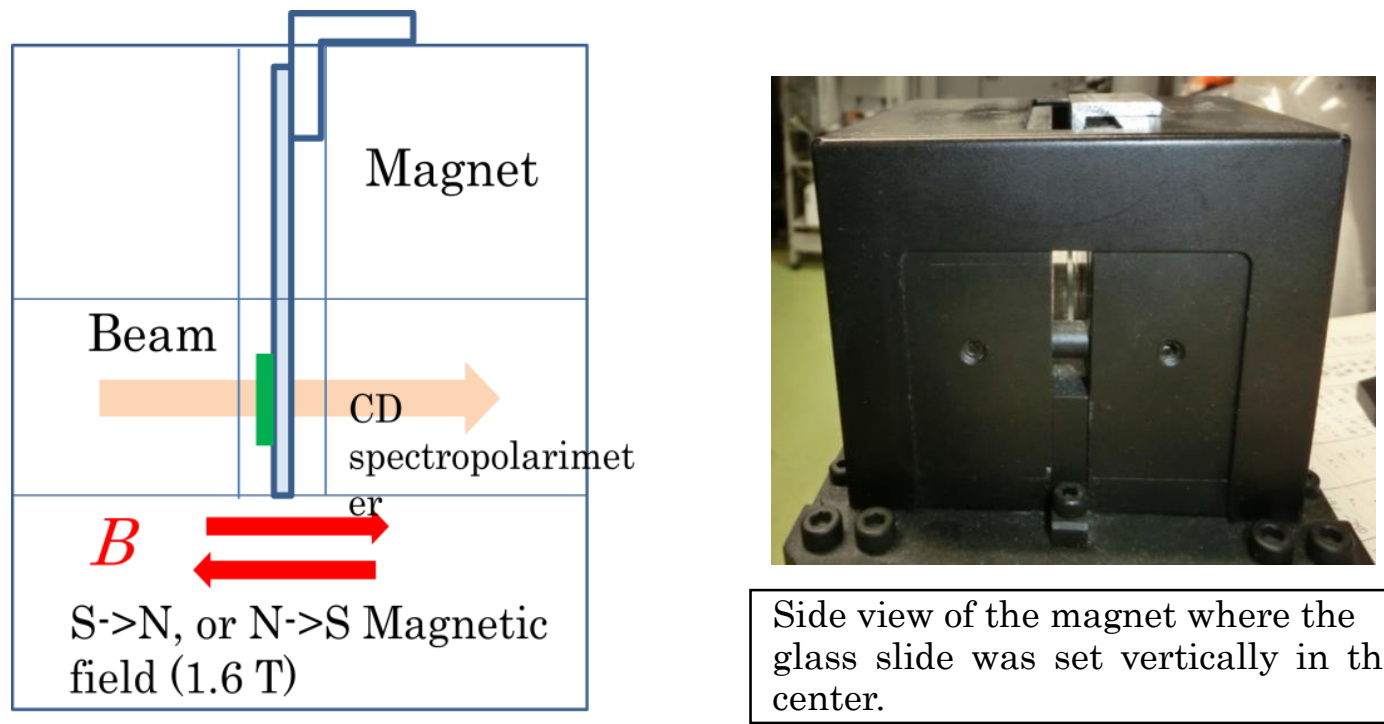

Side view of the magnet where the glass slide was set vertically in the center.

Figure S-1 Schematic illustration of the permanent magnet (PM491, 1.6 T, Jasco) used for the measurement of ICD and MCD spectra of silica gel slide sample. 
2. Comparison of the formation of J-aggregates of $\mathrm{H}_{4} \mathrm{TPP}^{2+}$ in the $\mathrm{o} / \mathrm{W}$ microemulsion and the silica gel systems.
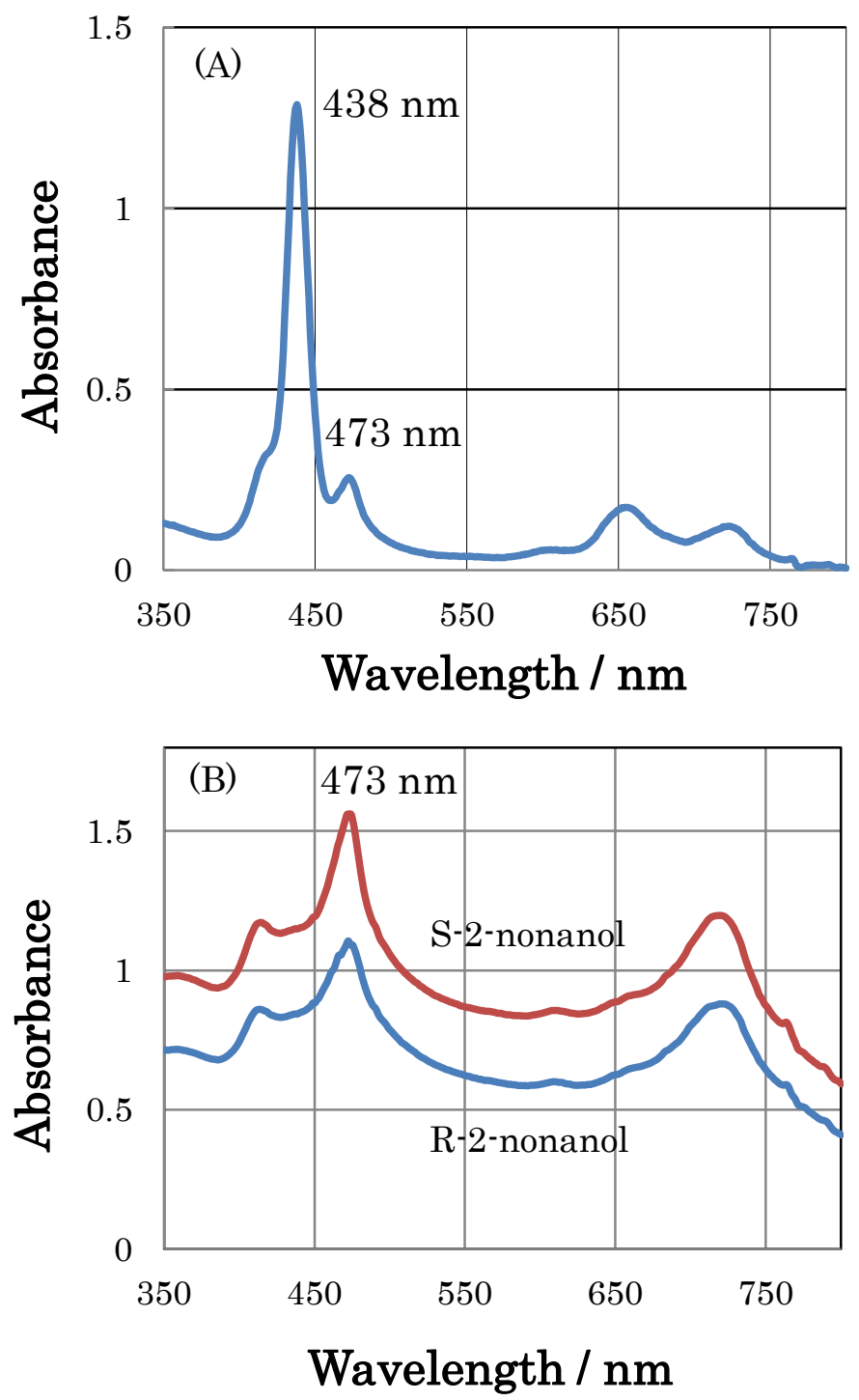

Figure S-2 Comparison of UV-Vis spectra between (A) o/w microemulsion and (B) silica gel plate; (A) $0.038 \mathrm{M} \mathrm{S}$ or R-2-nonanol, $5.0 \times 10^{-6} \mathrm{M} \mathrm{H}_{4} \mathrm{TPP}^{2+}$, 0.055M Triton X-100, 0.0157M toluene, 4M H $\mathrm{H}_{2} \mathrm{SO}_{4}$, (Watarai, H.; Hiramatsu, F.; Duc, H. T. T.; Lan, T. T. N., Chem. Lett. 2014, 43, 1257-1259). (B) Wakogel $+\mathrm{H}_{2} \mathrm{O}$ slurry $10 \mu \mathrm{L}, 0.11 \mathrm{M} \mathrm{R}$, S-2-nonanol $10 \mu \mathrm{L}, 4 \mathrm{M} \mathrm{H}_{2} \mathrm{SO}_{4} 10 \mu \mathrm{L}, 0.003 \mathrm{M}$ TPP $10 \mu \mathrm{L}$ 
3. Quantitative chiral recognition of 2-alkylalcohols was examined.
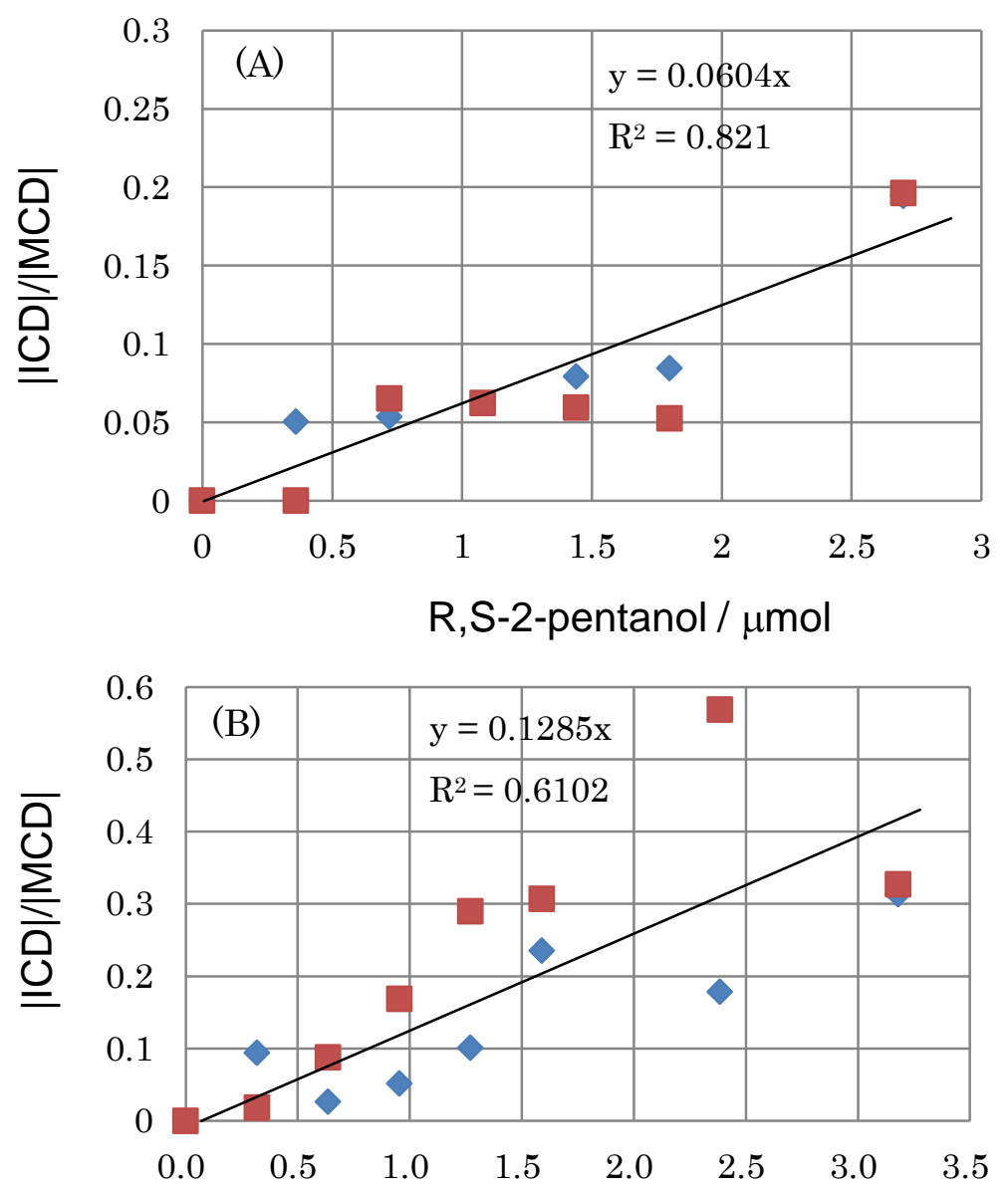

R.S-2-hexanol / umol

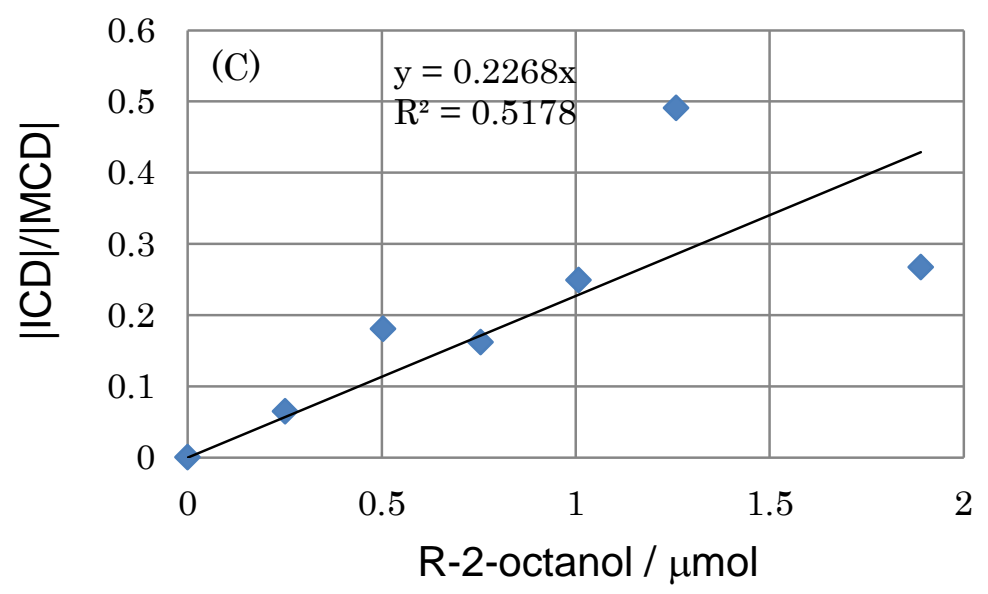

Figure S-3 The normalized ICD intensity of J-aggregates at the Q-band caused by the addition of chiral (A) R, S-2-pentanol, (B) R, S-2-hexanol and (C) R-2-octanol. 
4. Sensitivities of the chiral recognition depended on the alkyl chain length of the 2-alkylalsohols.

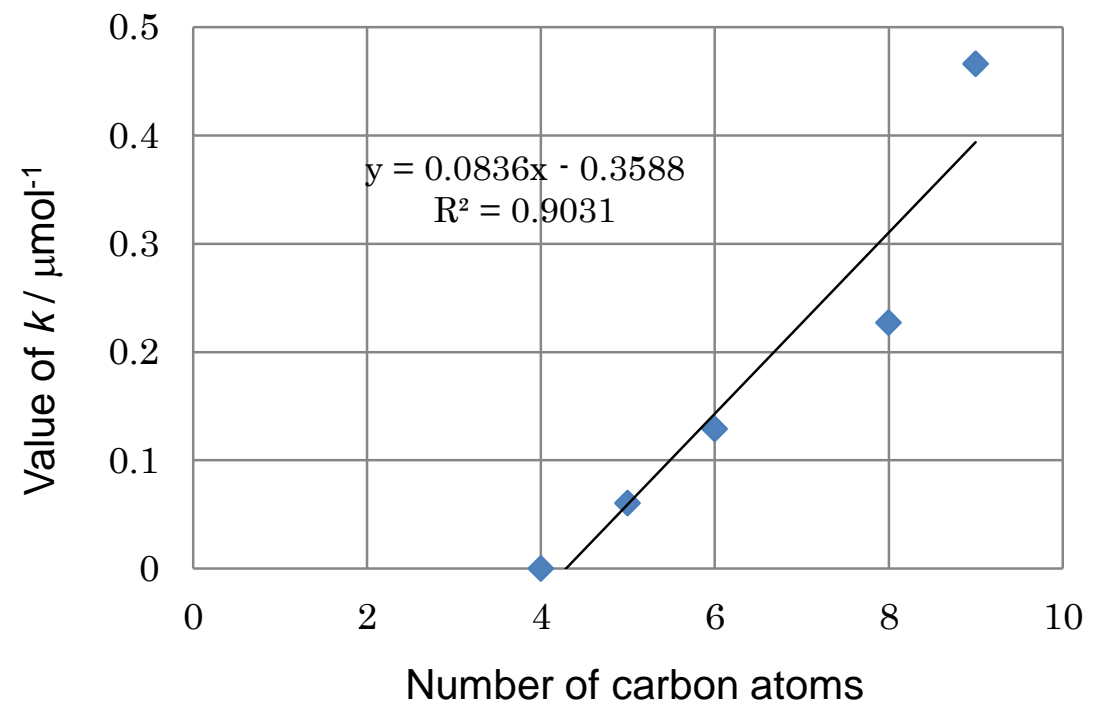

Figure S-4 Correlation between the values of sensitivity $(k)$ and the number of carbon atoms in the molecules of the 2-alkylalcohols. 